\title{
Backward Doubly Stochastic Differential Equations with Jumps and Stochastic Partial Differential-Integral Equations *
}

\author{
Qingfeng Zhu ${ }^{\mathrm{a}}$ and Yufeng $\mathrm{Shi}^{\mathrm{b} \dagger}$ \\ a School of Statistics and Mathematics, Shandong University of Finance, \\ Jinan 250014, China \\ ${ }^{\mathrm{b}}$ School of Mathematics, Shandong University, Jinan 250100, China
}

November 12, 2018

\begin{abstract}
In this paper, we study backward doubly stochastic differential equations driven by Brownian motions and Poisson process (BDSDEP in short) with non-Lipschitz coefficients on random time interval. The probabilistic interpretation for the solutions to a class of quasilinear stochastic partial differential-integral equations (SPDIEs in short) is the solutionBDSDEP. Under non-Lipschitz conditions, the existence and uniqueness results for measurable solutions of BDSDEP are established via the smoothing technique. Then, the continuous dependence for solutions of BDSDEP is derived. Finally, the probabilistic interpretation for the solutions to a class of quasilinear SPDIEs is given.

keywords: Backward doubly stochastic differential equations, stochastic partial differential-integral equations, random measure, Poisson process
\end{abstract}

\section{Introduction}

Nonlinear backward stochastic differential equations with Brownian motions as noise sources (BSDEs in short) have been independently introduced by Pardoux and Peng [7] and Duffie and Epstein [4. By virtue of BSDEs, Peng [9] has given a probabilistic interpretation (nonlinear Feynman-Kac formula) for the solutions of semilinear parabolic partial differential equations (PDEs in short). In [9, Peng also gave an existence and uniqueness result of BSDEs with random terminal time. And then Darling and Pardoux [3] proved an existence and uniqueness result for BSDEs with random terminal time under different assumptions. They applied their result to construct a continuous viscosity solution for a class of semilinear elliptic PDEs.

\footnotetext{
${ }^{*}$ This work is supported by National Natural Science Foundation of China Grant 10771122 , Natural Science Foundation of Shandong Province of China Grant Y2006A08 and National Basic Research Program of China (973 Program, No.2007CB814900)

${ }^{\dagger}$ Corresponding author, E-mail: yfshi@sdu.edu.cn
} 
A class of backward doubly stochastic differential equations (BDSDEs in short) was introduced by Pardoux and Peng [8] in 1994, in order to provide a probabilistic interpretation for the solutions of a class of semilinear stochastic partial differential equations (SPDEs in short). They have proved the existence and uniqueness of solutions for BDSDEs under uniformly Lipschitz conditions. Since then, Shi et al. [1] have relaxed the Lipschitz assumptions to linear growth conditions. Bally and Matoussi [1] have given a probabilistic interpretation of the solutions in Sobolev spaces for semilinear parabolic SPDEs in terms of BDSDEs. Zhang and Zhao [16] have proved the existence and uniqueness of solution for BDSDEs on infinite horizons, and described the stationary solutions of SPDEs by virtue of the solutions of BDSDEs on infinite horizons.

BSDEs driven by Brownian motions and Poisson process (BSDEP in short) were first discussed by Tang and Li [14]. After then Situ [12] obtained an existence and uniqueness result for BSDEP with non-Lipschitz coefficients, so as to give a probabilistic interpretation for solutions of partial differential-integral equations (PDIEs in short). Barles et al. [2] and Yin and Mao [15] discussed viscosity solutions for a system of partial differential-integral equations in terms of BSDEs with jumps. Recently BDSDEs driven by Brownian motions and Poisson process (BDSDEP in short) with Lipschitzian coefficients on a fixed time interval were discussed by Sun and Lu [13].

Because of their important significance to SPDEs, it is necessary to give intensive investigation to the theory of BDSDEs. In this paper we study BDSDEs driven by Brownian motions and Poisson process (BDSDEP in short) with non-Lipschitzian coefficients on random time interval. Here the coefficients are assumed to be weaker than linear growth, continuous and to satisfy some weak "monotone" condition. BDSDEP can provide more extensive frameworks for the probabilistic interpretations (so-called nonlinear stochastic Feynman-Kac formula) for the solutions of a class of quasilinear stochastic partial differentialintegral equations (SPDIEs in short). First, We establish the existence and uniqueness results for measurable solutions of BDSDEP based on the smoothing technique. Then we discuss the continuous dependence for solutions of BDSDEP. Finally, by virtue of BDSDEP, we show the probabilistic interpretation for the solutions of a class of quasilinear SPDIEs.

The paper is organized as follows. In Section 2, the basic assumptions are given. In Section 3, the existence and uniqueness for BDSDEP with non-Lipschitz coefficients on random time interval is proved. In Section 4, the continuous dependence for solutions of BDSDEP is discussed. Finally, in Section 5, the probabilistic interpretation for the solutions to a class of quasilinear SPDIEs is given by virtue of this class of BDSDEP.

\section{Setting of the problem}

Let $(\Omega, \mathcal{F}, P)$ be a complete probability space, and $[0, T]$ be an arbitrarily large fixed time duration throughout this paper. We suppose $\left\{\mathcal{F}_{t}\right\}_{t \geq 0}$ is generated by the following three mutually independent processes: 
(i) Let $\left\{W_{t} ; 0 \leq t \leq T\right\}$ and $\left\{B_{t} ; 0 \leq t \leq T\right\}$ be two standard Brownian motions defined on $(\Omega, \mathcal{F}, P)$, with values respectively in $\mathbb{R}^{d}$ and in $\mathbb{R}^{l}$.

(ii) Let $N$ be a Poisson random measure, on $\mathbb{R}_{+} \times Z$, where $Z \subset \mathbb{R}^{r}$ is a nonempty open set equipped with its Borel field $\mathcal{B}(Z)$, with compensator $\widehat{N}(\mathrm{~d} z \mathrm{~d} t)=\lambda(\mathrm{d} z) \mathrm{d} t$, such that $\widetilde{N}(A \times[0, t])=(N-\widehat{N})(A \times[0, t])_{t \geq 0}$ is a martingale for all $A \in \mathcal{B}(Z)$ satisfying $\lambda(A)<\infty$. $\lambda$ is assumed to be a $\sigma$-finite measure on $(Z, \mathcal{B}(Z))$ and is called the characteristic measure.

Let $\mathcal{N}$ denote the class of $P$-null elements of $\mathcal{F}$. For each $t \in[0, T]$, we define $\mathcal{F}_{t} \doteq \mathcal{F}_{t}^{W} \vee \mathcal{F}_{t, T}^{B} \vee \mathcal{F}_{t}^{N}$, where for any process $\left\{\eta_{t}\right\}, \mathcal{F}_{s, t}^{\eta}=\sigma\left\{\eta_{r}-\eta_{s} ; s \leq r \leq t\right\} \vee \mathcal{N}$, $\mathcal{F}_{t}^{\eta}=\mathcal{F}_{0, t}^{\eta}$. Note that the collection $\left\{\mathcal{F}_{t}, t \in[0, T]\right\}$ is neither increasing nor decreasing, and it does not constitute a classical filtration.

Let $\tau=\{\tau(\omega)\}$ be an $\mathcal{F}_{t}$-measurable time on $[0, T]$. We introduce the following notations:

$$
\begin{aligned}
& S^{2}\left([0, \tau] ; \mathbb{R}^{n}\right)=\left\{v_{t}, 0 \leq t \leq \tau \text {, is an } \mathbb{R}^{n} \text {-valued, } \mathcal{F}_{t^{-}}\right. \text {-measurable process } \\
& \text { such that } \left.E\left(\sup _{0 \leq t \leq \tau}\left|v_{t}\right|^{2}\right)<\infty\right\} \text {, } \\
& M^{2}\left(0, \tau ; \mathbb{R}^{n}\right)=\left\{v_{t}, 0 \leq t \leq \tau \text {, is an } \mathbb{R}^{n} \text {-valued, } \mathcal{F}_{t}\right. \text {-measurable process } \\
& \text { such that } \left.E \int_{0}^{\tau}\left|v_{t}\right|^{2} d t<\infty\right\} \text {, } \\
& F_{N}^{2}\left(0, \tau ; \mathbb{R}^{n}\right)=\left\{k_{t}, 0 \leq t \leq \tau \text {, is an } \mathbb{R}^{n} \text {-valued, } \mathcal{F}_{t}\right. \text {-measurable process } \\
& \text { such that } \left.E \int_{0}^{\tau} \int_{Z}\left|k_{t}(z)\right|^{2} \lambda(d z) d t<\infty\right\} \text {, } \\
& L_{\lambda(\cdot)}^{2}\left(\mathbb{R}^{n}\right)=\left\{k(z), k(z) \text { is an } \mathbb{R}^{n} \text {-valued, } \mathcal{B}(Z)\right. \text {-measurable function } \\
& \text { such that } \left.\|k\|=\left(\int_{Z}|k(z)|^{2} \lambda(d z)\right)^{1 / 2}<\infty\right\} \text {, } \\
& L^{2}\left(\Omega, \mathcal{F}_{\tau}, P ; \mathbb{R}^{n}\right)=\left\{\xi, \xi \text { is an } \mathbb{R}^{n} \text {-valued, } \mathcal{F}_{\tau}\right. \text {-measurable random variable } \\
& \text { such that } \left.E|\xi|^{2}<\infty\right\} \text {. }
\end{aligned}
$$

Consider the following BDSDE with Brownian motions and Poisson Process (BDSDEP in short):

$$
\begin{aligned}
P_{t}= & \xi+\int_{t \wedge \tau}^{\tau} f\left(s, P_{s}, Q_{s}, K_{s}\right) \mathrm{d} s+\int_{t \wedge \tau}^{\tau} g\left(s, P_{s}, Q_{s}, K_{s}\right) d B_{s} \\
& -\int_{t \wedge \tau}^{\tau} Q_{s} d W_{s}-\int_{t \wedge \tau}^{\tau} \int_{Z} K_{s}(z) \widetilde{N}(d z d s), \quad t \geq 0,
\end{aligned}
$$

where $\xi \in L^{2}\left(\Omega, \mathcal{F}_{\tau}, P ; \mathbb{R}^{n}\right)$,

$$
f: \Omega \times[0, T] \times \mathbb{R}^{n} \times \mathbb{R}^{n \times d} \times L_{\lambda(\cdot)}^{2}\left(\mathbb{R}^{n}\right) \rightarrow \mathbb{R}^{n},
$$

and

$$
g: \Omega \times[0, T] \times \mathbb{R}^{n} \times \mathbb{R}^{n \times d} \times L_{\lambda(\cdot)}^{2}\left(\mathbb{R}^{n}\right) \rightarrow \mathbb{R}^{n \times l} .
$$

We note that the integral with respect to $\left\{B_{t}\right\}$ is a "backward Itô integral" and the integral with respect to $\left\{W_{t}\right\}$ is a standard forward Itô integral. These two 
types of integrals are particular cases of the Itô-Skorohod integral (see Pardoux and Peng [8]). We use the usual inner product $\langle\cdot, \cdot\rangle$ and Euclidean norm $|\cdot|$ in $\mathbb{R}^{n}, \mathbb{R}^{n \times l}$ and $\mathbb{R}^{n \times d}$. All the equalities and inequalities mentioned in this paper are in the sense of $d t \times d P$ almost surely on $[0, \tau] \times \Omega$.

Definition 2.1 A solution of BDSDEP (11) is a triple of $\mathcal{F}_{t}$-measurable stochastic processes $(P, Q, K)$ which belongs to the space $S^{2}\left([0, \tau] ; \mathbb{R}^{n}\right) \times M^{2}\left(0, \tau ; \mathbb{R}^{n \times d}\right) \times$ $F_{N}^{2}\left(0, \tau ; \mathbb{R}^{n}\right)$ and satisfies BDSDEP (11).

We assume that

(H1) $\xi \in L^{2}\left(\Omega, \mathcal{F}_{\tau}, P ; \mathbb{R}^{n}\right)$;

(H2) $f(t, p, q, k), g(t, p, q, k)$ are continuous in $(p, q, k) \in \mathbb{R}^{n} \times \mathbb{R}^{n \times d} \times L_{\lambda(\cdot)}^{2}\left(\mathbb{R}^{n}\right)$;

(H3) $f=f_{1}+f_{2}, f_{i}=f_{i}(t, p, q, k): \Omega \times[0, T] \times \mathbb{R}^{n} \times \mathbb{R}^{n \times d} \times L_{\lambda(\cdot)}^{2}\left(\mathbb{R}^{n}\right) \rightarrow \mathbb{R}^{n}$, $i=1,2$, and $g(t, p, q, k)$ are $\mathcal{F}_{t}$-measurable processes, such that for all $t \in$ $[0, T] ; p, p_{1}, p_{2} \in \mathbb{R}^{n} ; q, q_{1}, q_{2} \in \mathbb{R}^{n \times d} ; k, k_{1}, k_{2} \in L_{\lambda(\cdot)}^{2}\left(\mathbb{R}^{n}\right)$,

$$
\begin{aligned}
& \left|f_{1}(t, p, q, k)\right| \leq \mu(t), \\
& \left|f_{2}(t, p, q, k)\right| \leq \mu(t)(1+|p|+|q|+\|k\|), \\
& |g(t, p, q, k)| \leq \mu(t)
\end{aligned}
$$

where $\mu(t) \geq 0$ is real and non-random function such that $\bar{\mu}=\int_{0}^{T} \mu^{2}(t) d t<$ $\infty$.

(H4) for all $t \in[0, T] ; p, p_{1}, p_{2} \in \mathbb{R}^{n} ; q, q_{1}, q_{2} \in \mathbb{R}^{n \times d} ; k, k_{1}, k_{2} \in L_{\lambda(\cdot)}^{2}\left(\mathbb{R}^{n}\right)$, such that

$$
\begin{aligned}
& \left\langle p_{1}-p_{2}, f_{1}\left(t, p_{1}, q_{1}, k_{1}\right)-f_{1}\left(t, p_{2}, q_{2}, k_{2}\right)\right\rangle \\
& \leq \mu\left(\rho\left(\left|p_{1}-p_{2}\right|^{2}\right)+\left|p_{1}-p_{2}\right|\left(\left|q_{1}-q_{2}\right|+\| k_{1}-k_{2} \mid\right)\right), \\
& \left|f_{1}\left(t, p, q, k_{1}\right)-f_{1}\left(t, p, q, k_{2}\right)\right| \leq \mu\left\|k_{1}-k_{2}\right\|, \\
& \left|f_{2}\left(t, p_{1}, q_{1}, k_{1}\right)-f_{2}\left(t, p_{2}, q_{2}, k_{2}\right)\right| \\
& \leq \mu\left(\left|p_{1}-p_{2}\right|+\left|q_{1}-q_{2}\right|+\left\|k_{1}-k_{2}\right\|\right), \\
& \left|g\left(t, p_{1}, q_{1}, k_{1}\right)-g\left(t, p_{2}, q_{2}, k_{2}\right)\right|^{2} \\
& \leq \mu\left(\left|p_{1}-p_{2}\right|^{2}+\left|p_{1}-p_{2}\right|\left(\left|q_{1}-q_{2}\right|+\| k_{1}-k_{2} \mid\right)\right),
\end{aligned}
$$

where $\mu>0$ is a constant, and $\rho(\cdot)$ is a nondecreasing, continuous and concave function from $R_{+}$to $R_{+}$such that $\rho(0)=0, \rho(u)>0$, as $u>0$, and $\int_{0^{+}} d u / \rho(u)=+\infty$.

\section{Existence and uniqueness of solutions to BDSDEP with non-Lipschitz coefficients}

In order to prove the existence and uniqueness results of solutions to BDSDEP with non-Lipschitz coefficients on random time interval, we introduce the following lemmas and theorems. 
Lemma 3.1 (A priori estimate). Under the assumption (H3). If $\left(P_{t}, Q_{t}, K_{t}\right)$ is a solution of (1), then

$$
E\left(\sup _{t \leq \tau}\left|P_{t}\right|^{2}+\int_{0}^{\tau}\left|Q_{t}\right|^{2} d t+\int_{0}^{\tau}\left\|K_{t}\right\|^{2} d t\right) \leq C_{T}<\infty
$$

where $C_{T} \geq 0$ is a constant depending on $T, \int_{0}^{T} \mu^{2}(t) d t$ and $E|\xi|^{2}$ only.

Proof. From (H3), we easily have

$$
\langle p, f(t, p, q, k)\rangle \leq \mu(t)\left(1+2|p|^{2}+|p|(|q|+\|k\|)\right),
$$

where $\mu(t)$ has the property stated in (H3). Applying Itô's formula to $\left|P_{t}\right|^{2}$, we have

$$
\begin{aligned}
& E\left(\left|P_{t \wedge \tau}\right|^{2}+\int_{t \wedge \tau}^{\tau}\left|Q_{s}\right|^{2} d s+\int_{t \wedge \tau}^{\tau}\left\|K_{s}\right\|^{2} d s\right) \\
= & E|\xi|^{2}+2 E \int_{t \wedge \tau}^{\tau}\left\langle P_{s}, f\left(s, P_{s}, Q_{s}, K_{s}\right)\right\rangle d s+E \int_{t \wedge \tau}^{\tau}\left|g\left(s, P_{s}, Q_{s}, K_{s}\right)\right|^{2} d s \\
\leq & E|\xi|^{2}+2 E \int_{t \wedge \tau}^{\tau} \mu(s)\left(1+2\left|P_{s}\right|^{2}+\left|P_{s}\right|\left(\left|Q_{s}\right|+\left\|K_{s}\right\|\right)\right) d s+\bar{\mu},
\end{aligned}
$$

we deduce

$$
\begin{aligned}
& E\left(\left|P_{t \wedge \tau}\right|^{2}+\frac{1}{2} \int_{t \wedge \tau}^{\tau}\left|Q_{s}\right|^{2} d s+\frac{1}{2} \int_{t \wedge \tau}^{\tau}\left\|K_{s}\right\|^{2} d s\right) \\
\leq & E|\xi|^{2}+T+2 \bar{\mu}+E \int_{t}^{T}\left(4 \mu(s)+2 \mu^{2}(s)\right)\left|P_{s}\right|^{2} d s .
\end{aligned}
$$

By Gronwall inequality, we have

$$
E\left(\left|P_{t \wedge \tau}\right|^{2}+\frac{1}{2} \int_{t \wedge \tau}^{\tau}\left|Q_{s}\right|^{2} d s+\frac{1}{2} \int_{t \wedge \tau}^{\tau}\left\|K_{s}\right\|^{2} d s\right) \leq \widetilde{C}_{T},
$$

where

$$
\widetilde{C}_{T}=\left(E|\xi|^{2}+T+2 \bar{\mu}\right) \exp \left(\int_{0}^{T}\left(4 \mu(s)+2 \mu^{2}(s)\right) d s\right) .
$$

In particular,

$$
E\left(\left|P_{0}\right|^{2}+\frac{1}{2} \int_{0}^{\tau}\left|Q_{s}\right|^{2} d s+\frac{1}{2} \int_{0}^{\tau}\left\|K_{s}\right\|^{2} d s\right) \leq \widetilde{C}_{T} .
$$

Applying Itô's formula to $\left|P_{t}\right|^{2}$ on $[0, t \wedge \tau]$, we have

$$
\begin{aligned}
& \left|P_{t \wedge \tau}\right|^{2} \\
= & \left|P_{0}\right|^{2}+2 \int_{0}^{t \wedge \tau}\left\langle P_{s}, f\left(s, P_{s}, Q_{s}, K_{s}\right)\right\rangle d s+2 \int_{0}^{t \wedge \tau}\left\langle P_{s}, g\left(s, P_{s}, Q_{s}, K_{s}\right)\right\rangle d B_{s} \\
& -2 \int_{0}^{t \wedge \tau}\left\langle P_{s}, Q_{s}\right\rangle d W_{s}+2 \int_{0}^{t \wedge \tau} \int_{Z}\left\langle P_{s}, K_{s}(z)\right\rangle \widetilde{N}(d z d s) \\
& +\int_{0}^{t \wedge \tau}\left|g\left(s, P_{s}, Q_{s}, K_{s}\right)\right|^{2} d s-\int_{0}^{t \wedge \tau}\left|Q_{s}\right|^{2} d s+\int_{0}^{t \wedge \tau}\left\|K_{s}\right\|^{2} d s .
\end{aligned}
$$


Taking supremum and expectation, we get

$$
\begin{aligned}
& E \sup _{t \leq \tau}\left|P_{t \wedge \tau}\right|^{2} \\
\leq & E\left|P_{0}\right|^{2}+2 E \int_{0}^{\tau} \mu(s)\left(1+2\left|P_{s}\right|^{2}+\left|P_{s}\right|\left(\left|Q_{s}\right|+\left\|K_{s}\right\|\right)\right) d s+\int_{0}^{T} \mu^{2}(s) d s \\
& +E \int_{0}^{\tau}\left|Q_{s}\right|^{2} d s+E \int_{0}^{\tau}|| K_{s} \|^{2} d s+2 E \sup _{t \leq \tau}\left|\int_{0}^{t \wedge \tau}\left\langle P_{s}, g\left(s, P_{s}, Q_{s}, K_{s}\right)\right\rangle d B_{s}\right| \\
& +2 E \sup _{t \leq \tau}\left|\int_{0}^{t \wedge \tau}\left\langle P_{s}, Q_{s}\right\rangle d W_{s}\right|+2 E \sup _{t \leq \tau}\left|\int_{0}^{t \wedge \tau} \int_{Z}\left\langle P_{s}, K_{s}(z)\right\rangle \widetilde{N}(d z d s)\right| .
\end{aligned}
$$

By Burkholder-Davis-Gundy's inequality, we deduce

$$
\begin{aligned}
& E\left(\sup _{t \leq \tau}\left|\int_{0}^{t \wedge \tau}\left\langle P_{s}, g\left(s, P_{s}, Q_{s}, K_{s}\right)\right\rangle d B_{s}\right|\right) \\
\leq & c E\left(\int_{0}^{\tau}\left|P_{s \wedge \tau}\right|^{2} \cdot\left|g\left(s, P_{s}, Q_{s}, K_{s}\right)\right|^{2} d s\right)^{1 / 2} \\
\leq & c E\left(\left(\sup _{t \leq \tau}\left|P_{t \wedge \tau}\right|^{2}\right)^{1 / 2}\left(\int_{0}^{\tau}\left|g\left(s, P_{s}, Q_{s}, K_{s}\right)\right|^{2} d s\right)^{1 / 2}\right) \\
\leq & \frac{1}{8} E \sup _{t \leq \tau}\left|P_{t \wedge \tau}\right|^{2}+2 c^{2} \int_{0}^{T} \mu^{2}(s) d s .
\end{aligned}
$$

In the same way, we have

$$
\begin{aligned}
& E \sup _{t \leq \tau}\left|\int_{0}^{t \wedge \tau}\left\langle P_{s}, Q_{s}\right\rangle d W_{s}\right| \leq \frac{1}{8} E \sup _{t \leq \tau}\left|P_{t \wedge \tau}\right|^{2}+2 c^{2} \int_{0}^{\tau}\left|Q_{s}\right|^{2} d s \\
& E \sup _{t \leq \tau}\left|\int_{0}^{t \wedge \tau} \int_{Z}\left\langle P_{s}, K_{s}(z)\right\rangle \tilde{N}(d z d s)\right| \leq \frac{1}{8} E \sup _{t \leq \tau}\left|P_{t \wedge \tau}\right|^{2}+2 c^{2} \int_{0}^{\tau}\left\|K_{s}\right\|^{2} d s .
\end{aligned}
$$

Hence

$$
\begin{aligned}
E \sup _{t \leq \tau}\left|P_{t \wedge \tau}\right|^{2} \leq & 4 E\left|P_{0}\right|^{2}+4 T+8 \bar{\mu}+4 E \int_{0}^{\tau}\left(4 \mu(s)+2 \mu^{2}(s)\right)\left|P_{s \wedge \tau}\right|^{2} d s \\
& +4\left(1+2 c^{2}\right) E \int_{0}^{\tau}\left(\left|Q_{s}\right|^{2}+\left\|K_{s}\right\|^{2}\right) d s \leq C_{T}<\infty
\end{aligned}
$$

As a preparation for the study of BDSDEP (11), we first discuss a simpler BDSDEP as follows

$$
\begin{aligned}
P_{t}= & \xi+\int_{t \wedge \tau}^{\tau} f(s) d s+\int_{t \wedge \tau}^{\tau} g(s) d B_{s}-\int_{t \wedge \tau}^{\tau} Q_{s} d W_{s} \\
& -\int_{t \wedge \tau}^{\tau} \int_{Z} K_{s}(z) \widetilde{N}(d z d s), \quad t \geq 0 .
\end{aligned}
$$

We have 
Lemma 3.2 Given $\xi \in L^{2}\left(\Omega, \mathcal{F}_{\tau}, P ; \mathbb{R}^{n}\right), f(t) \in M^{2}\left(0, \tau ; \mathbb{R}^{n}\right)$ and $g(t) \in M^{2}(0$, $\left.\tau ; \mathbb{R}^{n \times l}\right)$, then (20) has a unique solution in $S^{2}\left([0, \tau] ; \mathbb{R}^{n}\right) \times M^{2}\left(0, \tau ; \mathbb{R}^{n \times d}\right) \times$ $F_{N}^{2}\left(0, \tau ; \mathbb{R}^{n}\right)$.

Proof. Uniqueness. Let $\left(P^{1}, Q^{1}\right)$ and $\left(P^{2}, Q^{2}\right)$ be two solution of (2). Applying Itô's formula to $\left|P_{t}^{1}-P_{t}^{2}\right|^{2}$, we have

$$
E\left|P_{t \wedge \tau}^{1}-P_{t \wedge \tau}^{2}\right|^{2}+E \int_{t \wedge \tau}^{\tau}\left|Q_{s}^{1}-Q_{s}^{2}\right|^{2} d s+E \int_{t \wedge \tau}^{\tau}\left\|K_{s}^{1}-K_{s}^{2}\right\|^{2} d s=0 .
$$

Then

$$
E\left|P_{t \wedge \tau}^{1}-P_{t \wedge \tau}^{2}\right|^{2}=0, E \int_{t \wedge \tau}^{\tau}\left|Q_{s}^{1}-Q_{s}^{2}\right|^{2} d s=0
$$

and

$$
E \int_{t \wedge \tau}^{\tau}\left\|K_{s}^{1}-K_{s}^{2}\right\|^{2} d s=0,0 \leq t \leq T .
$$

Hence $P_{t}^{1}=P_{t}^{2}, Q_{t}^{1}=Q_{t}^{2}$ and $K_{t}^{1}=K_{t}^{2}$ a.s.. The uniqueness is obtained.

Existence. We define the filtration $\left(\mathcal{G}_{t}\right)_{0 \leq t \leq T}$ by

$$
\mathcal{G}_{t}=\mathcal{F}_{t}^{W} \vee \mathcal{F}_{T}^{B} \vee \mathcal{F}_{t}^{N}
$$

and the $\mathcal{G}_{t}$-square integrable martingale

$$
M_{t}=E\left[\xi+\int_{0}^{\tau} f(s) d s+\int_{0}^{\tau} g(s) d B_{s} \mid \mathcal{G}_{t}\right], \quad t \geq 0 .
$$

An obvious extension of Itô's martingale representation theorem (see [6]) yields the existence of $\left(Q_{s}, K_{s}\right)$ such that

$$
M_{t}=M_{0}+\int_{0}^{t} Q_{s} d W_{s}+\int_{0}^{t} \int_{Z} K_{s}(z) \tilde{N}(d z d s),
$$

and

$$
E \int_{0}^{T}\left(\left|Q_{s}\right|^{2}+\left\|K_{s}\right\|^{2}\right) d s<\infty
$$

Particularly

$$
\begin{aligned}
& M_{0}+\int_{0}^{\tau} Q_{s} d W_{s}+\int_{0}^{\tau} \int_{Z} K_{s}(z) \tilde{N}(d z d s) \\
= & M_{\tau}=\xi+\int_{0}^{\tau} f(s) d s+\int_{0}^{\tau} g(s) d B_{s}
\end{aligned}
$$

or

$$
\begin{aligned}
& M_{0}+\int_{0}^{t \wedge \tau} Q_{s} d W_{s}+\int_{0}^{t \wedge \tau} \int_{Z} K_{s}(z) \widetilde{N}(d z d s) \\
= & \xi+\int_{0}^{\tau} f(s) d s+\int_{0}^{\tau} g(s) d B_{s}-\int_{t \wedge \tau}^{\tau} Q_{s} z d W_{s}-\int_{t \wedge \tau}^{\tau} \int_{Z} K_{s}(z) \widetilde{N}(d z d s) .
\end{aligned}
$$


We set

$$
P_{t}=E\left[\xi+\int_{t \wedge \tau}^{\tau} f(s) d s+\int_{t \wedge \tau}^{\tau} g(s) d B_{s} \mid \mathcal{G}_{t}\right], \quad t \geq 0 .
$$

It follows that

$$
\begin{aligned}
P_{t}= & E\left[\xi+\int_{0}^{\tau} f(s) d s+\int_{0}^{\tau} g(s) d B_{s} \mid \mathcal{G}_{t}\right] \\
& -E\left[\int_{0}^{t \wedge \tau} f(s) d s+\int_{0}^{t \wedge \tau} g(s) d B_{s} \mid \mathcal{G}_{t}\right] \\
= & M_{0}+\int_{0}^{t \wedge \tau} Q_{s} d W_{s}+\int_{0}^{t \wedge \tau} \int_{Z} K_{s}(z) \tilde{N}(d z d s) \\
& -\int_{0}^{t \wedge \tau} f(s) d s-\int_{0}^{t \wedge \tau} g(s) d B_{s} .
\end{aligned}
$$

This with (3) implies that $\left(P_{t}, Q_{t}, K_{t}\right)$ solves (2).

In the following of this section we derive the existence and uniqueness results for solutions of BDSDEP on random time interval with Lipschitzian and nonLipschitzian coefficients. The first one, that is Theorem 3.3. deal with the case where $f$ is Lipschitz continuous.

Theorem 3.3 Under the assumptions (H1)-(H4), if $f_{1}=0$, (1)has a unique solution $\left(P_{t}, Q_{t}, K_{t}\right)$ in $S^{2}\left([0, \tau] ; \mathbb{R}^{n}\right) \times M^{2}\left(0, \tau ; \mathbb{R}^{n \times d}\right) \times F_{N}^{2}\left(0, \tau ; \mathbb{R}^{n}\right)$.

Proof. We define recursively a sequence $\left\{\left(P_{t}^{i}, Q_{t}^{i}, K_{t}^{i}\right)\right\}_{i=0,1, \ldots}$ as follows. Let $P_{t}^{0}=0, Q_{t}^{0}=0, K_{t}^{0}=0$. By Lemma 3.2, for any $\left(P_{t}^{i}, Q_{t}^{i}, K_{t}^{i}\right) \in S^{2}\left([0, \tau] ; \mathbb{R}^{n}\right) \times$ $M^{2}\left(0, \tau ; \mathbb{R}^{n \times d}\right) \times F_{N}^{2}\left(0, \tau ; \mathbb{R}^{n}\right), i=1,2$ there exists unique $\left(P_{t}^{i+1}, Q_{t}^{i+1}, K_{t}^{i+1}\right)$, satisfying

$$
\begin{aligned}
P_{t}^{i+1}= & \xi+\int_{t \wedge \tau}^{\tau} f\left(s, \bar{P}_{s}^{i}, \bar{Q}_{s}^{i}, \bar{K}_{s}^{i}\right) d s+\int_{t \wedge \tau}^{\tau} g\left(s, \bar{P}_{s}^{i}, \bar{Q}_{s}^{i}, \bar{K}_{s}^{i}\right) d B_{s} \\
& -\int_{t \wedge \tau}^{\tau} Q_{s}^{i+1} d W_{s}-\int_{t \wedge \tau}^{\tau} \int_{Z} K_{s}^{i+1}(z) \widetilde{N}(d z d s), t \geq 0 .
\end{aligned}
$$

Moreover, by Lemma 3.2. $\left(P_{t}^{i+1}, Q_{t}^{i+1}, K_{t}^{i+1}\right) \in S^{2}\left([0, \tau] ; \mathbb{R}^{n}\right) \times M^{2}\left(0, \tau ; \mathbb{R}^{n \times d}\right) \times$ $F_{N}^{2}\left(0, \tau ; \mathbb{R}^{n}\right)$.

Let $\bar{P}_{t}^{i+1}=P_{t}^{i+1}-P_{t}^{i}, \bar{Q}_{t}^{i+1}=Q_{t}^{i+1}-Q_{t}^{i}, \bar{K}_{t}^{i+1}=K_{t}^{i+1}-K_{t}^{i}$, By the Itô's formula to $\left|P_{t \wedge \tau}^{i+1}-P_{t \wedge \tau}^{i+1}\right|^{2} e^{-\beta t}$, we have

$$
\begin{aligned}
& E\left|P_{t \wedge \tau}^{1}-P_{t \wedge \tau}^{2}\right|^{2} e^{-\beta t}+\beta E \int_{t \wedge \tau}^{\tau}\left|P_{s}^{1}-P_{s}^{2}\right|^{2} e^{-\beta s} d s \\
& +E \int_{t \wedge \tau}^{\tau}\left|Q_{s}^{1}-Q_{s}^{2}\right|^{2} e^{-\beta s} d s+E \int_{t \wedge \tau}^{\tau}\left\|K_{s}^{1}-K_{s}^{2}\right\|^{2} e^{-\beta s} d s \\
= & 2 E \int_{t \wedge \tau}^{\tau}\left\langle P_{s}^{1}-P_{s}^{2}, f\left(s, \bar{P}_{s}^{1}, \bar{Q}_{s}^{1}, \bar{K}_{s}^{1}\right)-f\left(s, \bar{P}_{s}^{2}, \bar{Q}_{s}^{2}, \bar{K}_{s}^{2}\right)\right\rangle e^{-\beta s} d s \\
& +E \int_{t \wedge \tau}^{\tau}\left|g\left(s, \bar{P}_{s}^{1}, \bar{Q}_{s}^{1}, \bar{K}_{s}^{1}\right)-g\left(s, \bar{P}_{s}^{2}, \bar{Q}_{s}^{2}, \bar{K}_{s}^{2}\right)\right|^{2} e^{-\beta s} d s
\end{aligned}
$$




$$
\begin{aligned}
\leq & 2 \mu E \int_{t \wedge \tau}^{\tau}\left|P_{s}^{1}-P_{s}^{2}\right|\left(\left|\bar{P}_{s}^{1}-\bar{P}_{s}^{2}\right|+\left|\bar{Q}_{s}^{1}-\bar{Q}_{s}^{2}\right|+\left\|\bar{K}_{s}^{1}-\bar{K}_{s}^{2}\right\|\right) e^{-\beta s} d s \\
& +\mu E \int_{t \wedge \tau}^{\tau}\left(\left|\bar{P}_{s}^{1}-\bar{P}_{s}^{2}\right|^{2}+\left|\bar{P}_{s}^{1}-\bar{P}_{s}^{2}\right|\left(\left|\bar{Q}_{s}^{1}-\bar{Q}_{s}^{2}\right|+\left\|\bar{K}_{s}^{1}-\bar{K}_{s}^{2}\right\|\right)\right) e^{-\beta s} d s \\
\leq & \frac{1}{4} E \int_{t \wedge \tau}^{\tau}\left(\left|\bar{P}_{s}^{1}-\bar{P}_{s}^{2}\right|^{2}+\left|\bar{Q}_{s}^{1}-\bar{Q}_{s}^{2}\right|^{2}+\left\|\bar{K}_{s}^{1}-\bar{K}_{s}^{2}\right\|^{2}\right) e^{-\beta s} d s \\
& +12 \mu E \int_{t \wedge \tau}^{\tau}\left|P_{s}^{1}-P_{s}^{2}\right|^{2} e^{-\beta s} d s+3 \mu E \int_{t \wedge \tau}^{\tau}\left|\bar{P}_{s}^{1}-\bar{P}_{s}^{2}\right|^{2} e^{-\beta s} d s \\
& +\frac{1}{4} E \int_{t \wedge \tau}^{\tau}\left(\left|\bar{Q}_{s}^{1}-\bar{Q}_{s}^{2}\right|^{2}+\left\|\bar{K}_{s}^{1}-\bar{K}_{s}^{2}\right\|^{2}\right) e^{-\beta s} d s,
\end{aligned}
$$

we deduce

$$
\begin{aligned}
& E\left|P_{t \wedge \tau}^{1}-P_{t \wedge \tau}^{2}\right|^{2} e^{-\beta t}+(\beta-12 \mu) E \int_{t \wedge \tau}^{\tau}\left|P_{s}^{1}-P_{s}^{2}\right|^{2} e^{-\beta s} d s \\
& +E \int_{t \wedge \tau}^{\tau}\left|Q_{s}^{1}-Q_{s}^{2}\right|^{2} e^{-\beta s} d s+E \int_{t \wedge \tau}^{\tau}\left\|K_{s}^{1}-K_{s}^{2}\right\|^{2} e^{-\beta s} d s \\
\leq & \frac{1}{2} E \int_{t \wedge \tau}^{\tau}\left(\left|\bar{Q}_{s}^{1}-\bar{Q}_{s}^{2}\right|^{2}+\left\|\bar{K}_{s}^{1}-\bar{K}_{s}^{2}\right\|^{2}\right) e^{-\beta s} d s \\
& +\left(\frac{1}{4}+3 \mu\right) E \int_{t \wedge \tau}^{\tau}\left|\bar{P}_{s}^{1}-\bar{P}_{s}^{2}\right|^{2} e^{-\beta s} d s,
\end{aligned}
$$

Now choose $\beta=12 \mu+\frac{1+12 \mu}{2}$, and define $\bar{c}=\frac{1+12 \mu}{2}$.

$$
\begin{aligned}
& E\left|P_{t \wedge \tau}^{1}-P_{t \wedge \tau}^{2}\right|^{2} e^{-\beta t} \\
& +\int_{t \wedge \tau}^{\tau}\left(\bar{c}\left|P_{s}^{1}-P_{s}^{2}\right|^{2}+\left|Q_{s}^{1}-Q_{s}^{2}\right|^{2}+\left\|K_{s}^{1}-K_{s}^{2}\right\|^{2}\right) e^{-\beta s} d s \\
\leq & \frac{1}{2} E \int_{t \wedge \tau}^{\tau}\left(\bar{c}\left|\bar{P}_{s}^{1}-\bar{P}_{s}^{2}\right|^{2}+\left|\bar{Q}_{s}^{1}-\bar{Q}_{s}^{2}\right|^{2}+\left\|\bar{K}_{s}^{1}-\bar{K}_{s}^{2}\right\|^{2}\right) e^{-\beta s} d s,
\end{aligned}
$$

It follows immediately that

$$
\begin{gathered}
E \int_{t \wedge \tau}^{\tau}\left(\bar{c}\left|P_{s}^{1}-P_{s}^{2}\right|^{2}+\left|Q_{s}^{1}-Q_{s}^{2}\right|^{2}+\left\|K_{s}^{1}-K_{s}^{2}\right\|^{2}\right) e^{-\beta s} d s \\
\leq \frac{1}{2} E \int_{t \wedge \tau}^{\tau}\left(\bar{c}\left|\bar{P}_{s}^{1}-\bar{P}_{s}^{2}\right|^{2}+\left|\bar{Q}_{s}^{1}-\bar{Q}_{s}^{2}\right|^{2}+\left\|\bar{K}_{s}^{1}-\bar{K}_{s}^{2}\right\|^{2}\right) e^{-\beta s} d s,
\end{gathered}
$$

and $\left\{\left(P_{t}^{i}, Q_{t}^{i}, K_{t}^{i}\right)\right\}_{i=0,1, \ldots}$ is a Cauchy sequence in $S^{2}\left([0, \tau] ; \mathbb{R}^{n}\right) \times M^{2}\left(0, \tau ; \mathbb{R}^{n \times d}\right) \times$ $F_{N}^{2}\left(0, \tau ; \mathbb{R}^{n}\right)$, and that

$$
\left\{\left(P_{t}, Q_{t}, K_{t}\right)\right\}=\lim _{i \rightarrow \infty}\left\{\left(P_{t}^{i}, Q_{t}^{i}, K_{t}^{i}\right)\right\}
$$

solves (1).

The next theorem is the main result of this section, which generalizes the result of Theorem 3.3 to the case where $f$ is continuous but not Lipschitz continuous.

Theorem 3.4 Under the assumptions (H1)-(H4), then (1)has a unique solution $\left(P_{t}, Q_{t}, K_{t}\right)$. 
Proof. Uniqueness. Let $\left(P_{s}^{1}, Q_{s}^{1}, K_{s}^{1}\right)$ and $\left(P_{s}^{2}, Q_{s}^{2}, K_{s}^{2}\right)$ be two solutions of (11). Applying Itô's formula to $\left|P_{s}^{1}-P_{s}^{2}\right|^{2}$, we obtain

$$
\begin{aligned}
& E\left(\left|P_{t \wedge \tau}^{1}-P_{t \wedge \tau}^{2}\right|^{2}+\int_{t \wedge \tau}^{\tau}\left|Q_{s}^{1}-Q_{s}^{2}\right|^{2} d s+\int_{t \wedge \tau}^{\tau}\left\|K_{s}^{1}-K_{s}^{2}\right\|^{2} d s\right) \\
= & 2 E \int_{t \wedge \tau}^{\tau}\left\langle P_{s}^{1}-P_{s}^{2}, f\left(s, P_{s}^{1}, Q_{s}^{1}, K_{s}^{1}\right)-f\left(s, P_{s}^{2}, Q_{s}^{2}, K_{s}^{2}\right)\right\rangle d s \\
& +E \int_{t \wedge \tau}^{\tau}\left|g\left(s, P_{s}^{1}, Q_{s}^{1}, K_{s}^{1}\right)-g\left(s, P_{s}^{2}, Q_{s}^{2}, K_{s}^{2}\right)\right|^{2} d s \\
\leq & 2 \mu E \int_{t \wedge \tau}^{\tau}\left(\rho\left(\left|P_{s}^{1}-P_{s}^{2}\right|^{2}\right)+\left|P_{s}^{1}-P_{s}^{2}\right|\left(\left|Q_{s}^{1}-Q_{s}^{2}\right|+\left\|K_{s}^{1}-K_{s}^{2}\right\|\right)\right) d s \\
& +\mu E \int_{t \wedge \tau}^{\tau}\left(\left|P_{s}^{1}-P_{s}^{2}\right|^{2}+\left|P_{s}^{1}-P_{s}^{2}\right|\left(\left|Q_{s}^{1}-Q_{s}^{2}\right|+\left\|K_{s}^{1}-K_{s}^{2}\right\|\right)\right) d s .
\end{aligned}
$$

From (H4), we have

$$
\begin{aligned}
X_{t} & =E\left(\left|P_{t \wedge \tau}^{1}-P_{t \wedge \tau}^{2}\right|^{2}+\frac{1}{2} \int_{t \wedge \tau}^{\tau}\left|Q_{s}^{1}-Q_{s}^{2}\right|^{2} d s+\frac{1}{2} \int_{t \wedge \tau}^{\tau}\left\|K_{s}^{1}-K_{s}^{2}\right\|^{2} d s\right) \\
& \leq \mu E \int_{t \wedge \tau}^{\tau}\left(2 \rho\left(\left|P_{s}^{1}-P_{s}^{2}\right|^{2}\right)+11\left|P_{s}^{1}-P_{s}^{2}\right|^{2}\right) d s \\
& \leq 11 \mu \int_{t}^{T} \rho_{1}\left(X_{s}\right) d s,
\end{aligned}
$$

where

$$
\rho_{1}(u)=2 \rho(u)+16 u .
$$

By the Bahari's inequality, we obtain

$$
E\left(\left|P_{t \wedge \tau}^{1}-P_{t \wedge \tau}^{2}\right|^{2}+\int_{t \wedge \tau}^{\tau}\left|Q_{s}^{1}-Q_{s}^{2}\right|^{2} d s+\int_{t \wedge \tau}^{\tau}\left\|K_{s}^{1}-K_{s}^{2}\right\|^{2} d s\right)=0
$$

for all $t \in[0, T]$.

It implies that for all $t \in[0, T]$

$$
E\left|P_{t \wedge \tau}^{1}-P_{t \wedge \tau}^{2}\right|^{2}=0, E \int_{0}^{\tau}\left|Q_{s}^{1}-Q_{s}^{2}\right|^{2} d s=0, E \int_{0}^{\tau}\left\|K_{s}^{1}-K_{s}^{2}\right\|^{2} d s=0 .
$$

Existence. For simplicity we assume that $f_{2}=0$. (In case $f_{2} \neq 0$ we can just smooth out $f_{1}$ and proceed as follows.) Let us smooth out $f$ to get $f^{n}$, i.e. let

$$
f^{n}(t, P, Q, K)=\int_{\mathbb{R}^{n+n \times l}} f\left(t, P-n^{-1} \bar{P}, Q-n^{-1} \bar{Q}, K\right) J(\bar{P}, \bar{Q}) d \bar{P} d \bar{Q},
$$

where $J(P, Q)=J_{1}(P) J_{2}(Q)$, and $J_{1}(P)$ is defined, for all $P \in \mathbb{R}^{n}$,

$$
J_{1}(P)=\left\{\begin{array}{cc}
c_{0} \exp \left(-\left(1-|P|^{2}\right)^{-1}\right), & \text { as }|P|<1, \\
0, & \text { otherwise }
\end{array}\right.
$$


such that the constant $c_{0}$ satisfies $\int_{\mathbb{R}^{n}} J(x) d x=1$. $J_{2}(Q)$ is similarly defined for any $Q \in \mathbb{R}^{n \times d}$. It easy to check that

$$
\begin{aligned}
& \left|f^{n}\left(t,, P_{1}, Q_{1}, K_{1}\right)-f^{n}\left(t, P_{2}, Q_{2}, K_{2}\right)\right| \\
\leq & C_{n} \mu\left(\left|P_{1}-P_{2}\right|+\left|Q_{1}-Q_{2}\right|+\left\|K_{1}-K_{2}\right\|\right),
\end{aligned}
$$

as $\left(P_{i}, Q_{i}, K_{i}\right) \in \mathbb{R}^{n} \times \mathbb{R}^{n \times d} \times L_{\lambda(\cdot)}^{2}\left(\mathbb{R}^{n}\right), i=1,2$. Hence by Theorem 3.3 , for each $n=1,2, \cdots$, there exists a unique solution $\left(P_{t}^{n}, Q_{t}^{n}, K_{t}^{n}\right)$ to solve the following BDSDEP

$$
\begin{aligned}
P_{t \wedge \tau}^{n}= & \xi+\int_{t \wedge \tau}^{\tau} f^{n}\left(s, P_{s}^{n}, Q_{s}^{n}, K_{s}^{n}\right) d s+\int_{t \wedge \tau}^{\tau} g\left(s, P_{s}^{n}, Q_{s}^{n}, K_{s}^{n}\right) d B_{s} \\
& -\int_{t \wedge \tau}^{\tau} Q_{s}^{n} d W_{s}-\int_{t \wedge \tau}^{\tau} \int_{Z} K_{s}^{n}(z) \tilde{N}(d z d s) .
\end{aligned}
$$

Applying Itô's formula to $\left|P_{t}^{n}-P_{t}^{m}\right|^{2}$, we have

$$
\begin{aligned}
& \left|P_{t \wedge \tau}^{n}-P_{t \wedge \tau}^{m}\right|^{2}+\int_{t \wedge \tau}^{\tau}\left|Q_{s}^{n}-Q_{s}^{m}\right|^{2} d s+\int_{t \wedge \tau}^{\tau}\left\|K_{s}^{n}-K_{s}^{m}\right\|^{2} d s \\
= & 2 \int_{t \wedge \tau}^{\tau}\left\langle P_{s}^{n}-P_{s}^{m}, f^{n}\left(s, P_{s}^{n}, Q_{s}^{n}, K_{s}^{n}\right)-f^{m}\left(s, P_{s}^{m}, Q_{s}^{m}, K_{s}^{m}\right)\right\rangle d s \\
& +\int_{t \wedge \tau}^{\tau}\left|g\left(s, P_{s}^{n}, Q_{s}^{n}, K_{s}^{n}\right)-g\left(s, P_{s}^{m}, Q_{s}^{m}, K_{s}^{m}\right)\right|^{2} d s \\
& -2 \int_{t \wedge \tau}^{\tau}\left\langle P_{s}^{n}-P_{s}^{m}, Q_{s}^{n}-Q_{s}^{m}\right\rangle d W_{s} \\
& +2 \int_{t \wedge \tau}^{\tau}\left\langle P_{s}^{n}-P_{s}^{m}, g\left(s, P_{s}^{n}, Q_{s}^{n}, K_{s}^{n}\right)-g\left(s, P_{s}^{m}, Q_{s}^{m}, K_{s}^{m}\right)\right\rangle d B_{s} \\
& -2 \int_{t \wedge \tau}^{\tau} \int_{Z}\left\langle P_{s}^{n}-P_{s}^{m}, K_{s}^{n}-K_{s}^{m}\right\rangle \widetilde{N}(d z d s)=\sum_{i=1}^{5} I_{i} .
\end{aligned}
$$

Note that

$$
\begin{aligned}
I_{1}= & 2 \int_{t \wedge \tau}^{\tau}\left\langle P_{s}^{n}-P_{s}^{m}, \int_{\mathbb{R}^{n+n \times l}}\left(f\left(s, P_{s}^{n}-n^{-1} \bar{P}, Q_{s}^{n}-n^{-1} \bar{Q}, K_{s}^{n}\right)\right.\right. \\
& \left.\left.-f\left(s, Y_{s}^{m}-m^{-1} \bar{P}, Z_{s}^{m}-m^{-1} \bar{Q}, K_{s}^{m}\right)\right) J(\bar{P}, \bar{Q}) d \bar{P} d \bar{Q}\right\rangle d s \\
\leq & \mu \int_{t \wedge \tau}^{\tau} \int_{\mathbb{R}^{n+n \times l}}\left(\left(\rho\left(\left|P_{s}^{n}-P_{s}^{m}-\left(n^{-1}-m^{-1}\right) \bar{P}\right|^{2}\right)\right.\right. \\
& +\left|P_{s}^{n}-P_{s}^{m}-\left(n^{-1}-m^{-1}\right) \bar{P}\right| \times\left(\left|Q_{s}^{n}-Q_{s}^{m}-\left(n^{-1}-m^{-1}\right) \bar{Q}\right|\right. \\
& \left.\left.\left.+\left\|K_{s}^{n}-K_{s}^{m}\right\|\right)\right)+\left|n^{-1}-m^{-1}\right||\bar{P}| 2\right) J(\bar{P}, \bar{Q}) d \bar{P} d \bar{Q} d s .
\end{aligned}
$$

Since by Lemma 3.1 for all $n$

$$
E\left(\sup _{t \leq \tau}\left|P_{t}^{n}\right|^{2}+\int_{0}^{\tau}\left|Q_{t}^{n}\right|^{2} d t+\int_{0}^{\tau}\left\|K_{t}^{n}\right\|^{2} d t\right) \leq C_{T}<\infty
$$


Hence

$$
\begin{aligned}
& E\left(\left|P_{t \wedge \tau}^{n}-P_{t \wedge \tau}^{m}\right|^{2}+\int_{t \wedge \tau}^{\tau}\left|Q_{s}^{n}-Q_{s}^{m}\right|^{2} d s+\int_{t \wedge \tau}^{\tau}\left\|K_{s}^{n}-K_{s}^{m}\right\|^{2} d s\right) \\
\leq & \bar{C}_{T}\left(\mu^{2}+\mu\right) \int_{t}^{T} \int_{\mathbb{R}^{n+n \times l}}\left(\rho\left(E\left|P_{s \wedge \tau}^{n}-P_{s \wedge \tau}^{m}-\left(n^{-1}-m^{-1}\right) \bar{P}\right|^{2}\right)\right. \\
& \left.+E\left|P_{s \wedge \tau}^{n}-P_{s \wedge \tau}^{m}\right|^{2}\right) J(\bar{P}, \bar{Q}) d \bar{P} d \bar{Q} d s+\bar{C}_{T}\left(n^{-1}+m^{-1}\right) .
\end{aligned}
$$

Note that

$$
\rho\left(2 E\left|Y_{s \wedge \tau}^{n}-Y_{s \wedge \tau}^{m}\right|^{2}+2\left(n^{-1}-m^{-1}\right)^{2}|\bar{P}|^{2}\right) \leq \rho\left(4 C_{T}+2|\bar{P}|^{2}\right) .
$$

But by assumption it yields that

$$
\int \rho\left(4 C_{T}+2|\bar{Y}|^{2}\right) J(\bar{P}, \bar{Q}) d \bar{P} d \bar{Q} \leq \rho\left(4 C_{T}+2\right)<\infty .
$$

Hence by Lemma 3.1 and by the Fatou lemma it is easily seen that

$$
\begin{aligned}
& \limsup _{n, m \rightarrow \infty} E\left|P_{t \wedge \tau}^{n}-P_{t \wedge \tau}^{m}\right|^{2}+\limsup _{n, m \rightarrow \infty} E \int_{t \wedge \tau}^{\tau}\left(\left|Q_{s}^{n}-Q_{s}^{m}\right|^{2}+\left\|K_{s}^{n}-K_{s}^{m}\right\|^{2}\right) d s \\
\leq & \widehat{C}_{T}\left(\mu^{2}+\mu\right) \int_{t}^{T} \rho_{1}\left(\limsup _{n, m \rightarrow \infty} 2 E\left|P_{s \wedge \tau}^{n}-P_{s \wedge \tau}^{m}\right|^{2}\right) d s,
\end{aligned}
$$

where $\rho_{1}(u)=\rho(u)+u$. By the Bahari's inequality, we obtain

$$
\limsup _{n, m \rightarrow \infty} E\left|P_{t \wedge \tau}^{n}-P_{t \wedge \tau}^{m}\right|^{2}=0, \text { for all } t \in[0, T],
$$

and

$$
\limsup _{n, m \rightarrow \infty} E \int_{0}^{\tau}\left(\left|Q_{s}^{n}-Q_{s}^{m}\right|^{2}+\left\|K_{s}^{n}-K_{s}^{m}\right\|^{2}\right) d s=0 .
$$

These, together with the Burkholder-Davis-Gundy's inequality, yield

$$
\lim _{n, m \rightarrow \infty} E \sup _{0 \leq t \leq \tau}\left|P_{t}^{n}-P_{t}^{m}\right|^{2}=0 .
$$

By the completeness of Banach space, we know that there exists a unique $(P$, $Q, K) \in S^{2}\left([0, \tau] ; \mathbb{R}^{n}\right) \times M^{2}\left(0, \tau ; \mathbb{R}^{n \times d}\right) \times F_{N}^{2}\left(0, \tau ; \mathbb{R}^{n}\right)$ such that as $n \rightarrow \infty$,

$$
\begin{aligned}
& E \sup _{0 \leq t \leq \tau}\left|P_{t}^{n}-P_{t}\right|^{2} \rightarrow 0, \\
& E \int_{0}^{\tau}\left|Q_{s}^{n}-Q_{s}\right|^{2} d s \rightarrow 0, \\
& E \int_{0}^{\tau}\left\|K_{s}^{n}-K_{s}\right\|^{2} d s \rightarrow 0 .
\end{aligned}
$$

Therefore we can take a subsequence $\left\{n_{k}\right\}$ of $\{n\}$, denote it by $\{n\}$ again such that almost surely for $(t, \omega) \in[0, T] \times \Omega$,

$$
\left(P_{t}^{n}, Q_{t}^{n}, K_{t}^{n}\right) \rightarrow\left(P_{t}, Q_{t}, K_{t}\right) \text { in } \mathbb{R}^{n} \times \mathbb{R}^{n \times l} \times L_{\lambda(\cdot)}\left(\mathbb{R}^{n}\right) .
$$


Hence by the continuity of $f$ in $(P, Q, K)$, (H3), Lemma 3.1 and the Lebesgue domination convergence theorem, we have that

$$
E \int_{0}^{\tau}\left|f^{n}\left(s, P_{s}^{n}, Q_{s}^{n}, K_{s}^{n}\right)-f\left(s, P_{s}, Q_{s}, K_{s}\right)\right| d s \rightarrow 0, n \rightarrow \infty .
$$

It is easy to check that $(P, Q, K)$ is a solution of (1) by taking the limit on both sides of (4).

\section{Continuous dependence for solutions of BDSDEP}

In this section, we discuss the continuous dependence for solutions of BDSDEP (11).

Theorem 4.1 For $m=0,1,2, \cdots$

(i) $f^{m}=f^{m}(t, p, q, k):[0, T] \times \mathbb{R}^{n} \times \mathbb{R}^{n \times d} \times L_{\lambda(\cdot)}^{2}\left(\mathbb{R}^{n}\right) \rightarrow \mathbb{R}^{n}$ are $\mathcal{F}_{t}$-measurable such that P-a.s.

$$
\left\langle p, f^{m}(t, p, q, k)\right\rangle \leq \mu(t)\left(1+|p|^{2}+|p|(|q|+|k|)\right),
$$

where $\mu(t)$ has the property stated in (H3);

(ii) for all $p_{1}, p_{2} \in \mathbb{R}^{n} ; q_{1}, q_{2} \in \mathbb{R}^{n \times d} ; k_{1}, k_{2} \in L_{\lambda(\cdot)}^{2}\left(\mathbb{R}^{n}\right)$, such that P-a.s.

$$
\begin{aligned}
& \left\langle p_{1}-p_{2}, f^{0}\left(t, p_{1}, q_{1}, k_{1}\right)-f^{0}\left(t, p_{2}, q_{2}, k_{2}\right)\right\rangle \\
\leq & \mu(t)\left(\rho\left(\left|p_{1}-p_{2}\right|^{2}\right)+\left|p_{1}-p_{2}\right|\left(\left|q_{1}-q_{2}\right|+\left|k_{1}-k_{2}\right|\right)\right),
\end{aligned}
$$

where $\rho(\cdot)$ has the property stated in $\left(\mathrm{H}_{4}\right)$;

(iii) $g^{m}=g^{m}(t, p, q, k):[0, T] \times \mathbb{R}^{n} \times \mathbb{R}^{n \times d} \times L_{\lambda(\cdot)}^{2}\left(\mathbb{R}^{n}\right) \rightarrow \mathbb{R}^{n \times l}$ are $\mathcal{F}_{t}$-measurable such that $P$-a.s.

$$
\begin{aligned}
& \left|g^{m}(t, p, q, k)\right| \leq \mu(t), \\
& \left|g^{m}\left(t, p_{1}, q_{1}, k_{1}\right)-g^{m}\left(t, p_{2}, q_{2}, k_{2}\right)\right|^{2} \\
\leq & \mu(t)\left(\left|p_{1}-p_{2}\right|^{2}+\left|p_{1}-p_{2}\right|\left(\left|q_{1}-q_{2}\right|+\left|k_{1}-k_{2}\right|\right)\right),
\end{aligned}
$$

where $\mu(t)$ has the property stated in (H3);

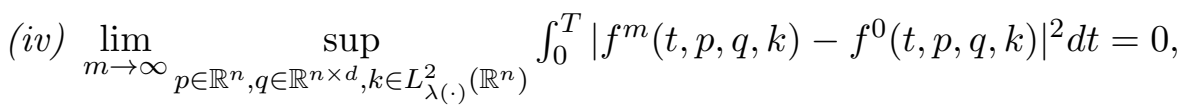

$$
\lim _{m \rightarrow \infty} \sup _{p \in \mathbb{R}^{n}, q \in \mathbb{R}^{n \times d}, k \in L_{\lambda(\cdot)}^{2}\left(\mathbb{R}^{n}\right)} \int_{0}^{T}\left|g^{m}(t, p, q, k)-g^{0}(t, p, q, k)\right|^{2} d t=0 .
$$

(v) $\xi^{m}$ is $\mathcal{F}_{\tau}$-measurable and

$$
E\left|\xi^{m}-\xi^{0}\right|^{2} \rightarrow 0, \text { as } m \rightarrow \infty, E\left|\xi^{m}\right|^{2}<\infty .
$$


If $\left(P_{t}^{m}, Q_{t}^{m}, K_{t}^{m}\right)$ are solutions of the following BDSDEP: as $0 \leq s \leq T$

$$
\begin{aligned}
P_{s \wedge \tau}^{m}= & \xi^{m}+\int_{s \wedge \tau}^{T \wedge \tau} f^{m}\left(s, P_{r}^{m}, Q_{r}^{m}, K_{r}^{m}\right) \mathrm{d} r+\int_{s \wedge \tau}^{T \wedge \tau} g^{m}\left(s, P_{r}^{m}, Q_{r}^{m}, K_{r}^{m}\right) d B_{r} \\
& -\int_{s \wedge \tau}^{T \wedge \tau} Q_{r}^{m} \mathrm{~d} W_{r}-\int_{s \wedge \tau}^{T \wedge \tau} \int_{Z} K_{r}^{m}(z) \tilde{N}(d z d r), \quad m=0,1,2, \cdots,
\end{aligned}
$$

then for all $0 \leq s \leq T$

$$
\lim _{m \rightarrow \infty} E\left(\sup _{s \leq r \leq T}\left|P_{r \wedge \tau}^{m}-P_{r \wedge \tau}^{0}\right|^{2}+\int_{s \wedge \tau}^{T \wedge \tau}\left(\left|Q_{r}^{m}-Q_{r}^{0}\right|^{2}+\left\|K_{r}^{m}-K_{r}^{0}\right\|^{2}\right) d r\right)=0 .
$$

Proof. Applying Itô's formula to $\left|P_{s}^{m}-P_{s}^{0}\right|^{2}$, it follows that

$$
\begin{aligned}
& E\left(\left|P_{s \wedge \tau}^{m}-P_{s \wedge \tau}^{0}\right|^{2}+\int_{s \wedge \tau}^{T \wedge \tau}\left(\left|Q_{r}^{m}-Q_{r}^{0}\right|^{2}+\left\|K_{r}^{m}-K_{r}^{0}\right\|^{2}\right) d r\right) \\
\leq & C_{0}\left(\mu^{2}(s)+\mu(s)+1\right)\left(\int_{s}^{T} \rho_{1}\left(E\left|P_{s \wedge \tau}^{m}-P_{s \wedge \tau}^{0}\right|^{2}\right) d r+E\left|\xi^{m}-\xi^{0}\right|^{2}\right) \\
& +C_{0} E \int_{s}^{T}\left|f^{m}\left(P_{r}^{m}, Q_{r}^{m}, K_{r}^{m}\right)-f^{0}\left(P_{r}^{m}, Q_{r}^{m}, K_{r}^{m}\right)\right|^{2} d r \\
& +C_{0} E \int_{s}^{T}\left|g^{m}\left(P_{r}^{m}, Q_{r}^{m}, K_{r}^{m}\right)-g^{0}\left(P_{r}^{m}, Q_{r}^{m}, K_{r}^{m}\right)\right|^{2} d r
\end{aligned}
$$

where

$$
\rho_{1}(u)=\rho(u)+u
$$

Hence

$$
\limsup _{m \rightarrow \infty} E\left|P_{s \wedge \tau}^{m}-P_{s \wedge \tau}^{0}\right|^{2} \leq C_{0} \int_{s}^{T} \hat{\mu}(r) \rho_{1}\left(\limsup _{m \rightarrow \infty} E\left|P_{r \wedge \tau}^{m}-P_{r \wedge \tau}^{0}\right|^{2}\right) d r .
$$

By the Bahari's inequality, we obtain

$$
\limsup _{m \rightarrow \infty} E\left|P_{s \wedge \tau}^{m}-P_{s \wedge \tau}^{0}\right|^{2}=0 .
$$

It is easily derived that

$$
\lim _{m \rightarrow \infty} E\left(\int_{s \wedge \tau}^{T \wedge \tau}\left(\left|Q_{r}^{m}-Q_{r}^{0}\right|^{2}+\left\|K_{r}^{m}-K_{r}^{0}\right\|^{2}\right) d r\right)=0 .
$$


Applying Itô's formula to $\left|P_{s}^{m}-P_{s}^{0}\right|^{2}$ on $[0, t \wedge \tau]$, we have

$$
\begin{aligned}
& E \sup _{t \leq \tau}\left|P_{t \wedge \tau}^{m}-P_{t \wedge \tau}^{0}\right|^{2} \\
\leq & E\left|P_{0}^{m}-P_{0}^{0}\right|^{2}+\int_{0}^{\tau}\left|g^{m}\left(s, P_{s}^{m}, Q_{s}^{m}, K_{s}^{m}\right)-g^{0}\left(s, P_{s}^{0}, Q_{s}^{0}, K_{s}^{0}\right)\right|^{2} d s \\
& +2 E \int_{0}^{\tau}\left\langle P_{s}^{m}-P_{s}^{0}, f^{m}\left(s, P_{s}^{m}, Q_{s}^{m}, K_{s}^{m}\right)-f^{0}\left(s, P_{s}^{0}, Q_{s}^{0}, K_{s}^{0}\right)\right\rangle d s \\
& -\int_{0}^{\tau}\left|Q_{s}^{m}-Q_{s}^{0}\right|^{2} d s+\int_{0}^{\tau}\left\|K_{s}^{m}-K_{s}^{0}\right\|^{2} d s \\
& +2 E \sup _{t \leq \tau} \int_{0}^{t \wedge \tau}\left\langle P_{s}^{m}-P_{s}^{0}, g^{m}\left(s, P_{s}^{m}, Q_{s}^{m}, K_{s}^{m}\right)-g^{0}\left(s, P_{s}^{0}, Q_{s}^{0}, K_{s}^{0}\right)\right\rangle d B_{s} \\
& -2 E \sup _{t \leq \tau} \int_{0}^{t \wedge \tau}\left\langle P_{s}^{m}-P_{s}^{0}, Q_{s}^{m}-Q_{s}^{0}\right\rangle d W_{s} \\
& +2 E \sup _{t \leq \tau} \int_{0}^{t \wedge \tau} \int_{Z}\left\langle P_{s}^{m}-P_{s}^{0}, K_{s}^{m}(z)-K_{s}^{0}(z)\right\rangle \widetilde{N}(d z d s) .
\end{aligned}
$$

By the similar arguments in Lemma 3.1, we obtain

$$
\lim _{m \rightarrow \infty} E \sup _{s \leq r \leq T}\left|P_{r \wedge \tau}^{m}-P_{r \wedge \tau}^{0}\right|^{2}=0 .
$$

We also have the other useful continuous dependence for solutions of BDSDEP as follows:

Theorem 4.2 For $m=0,1,2, \cdots$

(i) $f^{m}(t, p, q, k)$ are $\mathcal{F}_{t}$-measurable such that P-a.s.

$$
\left|f^{m}(t, p, q, k)\right| \leq C_{0}(1+|p|+|q|+|k|),
$$

where $C_{0} \leq 0$ is a constant;

(ii) for all $p_{1}, p_{2} \in \mathbb{R}^{n} ; q_{1}, q_{2} \in \mathbb{R}^{n \times l} ; k_{1}, k_{2} \in \mathbb{R}^{n}$, such that

$$
\begin{aligned}
& \left\langle p_{1}-p_{2}, f^{m}\left(t, p_{1}, q_{1}, k_{1}\right)-f^{m}\left(t, p_{2}, q_{2}, k_{2}\right)\right\rangle \\
& \leq \mu(t) \rho\left(\left|p_{1}-p_{2}\right|^{2}\right)+\left|p_{1}-p_{2}\right|^{2}\left(\left|q_{1}-q_{2}\right|^{2}+\left|k_{1}-k_{2}\right|^{2}\right),
\end{aligned}
$$

where $\mu(t)$ has the property stated in (H3) and $\rho(\cdot)$ has the property stated in ( $\mathrm{H}_{4}$ );

(iii) The same as (iii) in Theorem 4.1;

(iv) $\lim _{m \rightarrow \infty} f^{m}(t, p, q, k)=f^{0}(t, p, q, k)$, P-a.s.

$\lim _{m \rightarrow \infty} g^{m}(t, p, q, k)=g^{0}(t, p, q, k), P$-a.s.

(v) The same as (v) in Theorem 4.1;

Then the conclusion of Theorem 4.1 still holds.

The proof can be completed similarly as that of Theorem 4.1, 


\section{The probabilistic interpretation of SPDIEs}

The connection of BDSDEs and systems of second-order quasilinear SPDEs was observed by Pardoux and Peng [8]. This can be regarded as a stochastic version of the well-known Feynman-Kac formula which gives a probabilistic interpretation for second-order SPDEs of parabolic types. Thereafter this subject has attracted many mathematicians, referred to Bally and Matoussi [1, Zhang and Zhao [16], $\mathrm{Hu}$ and Ren [5, see also Ren et al. [10]. In [5], the authors got a probabilistic interpretation for the solution of a semilinear SPDIE, via BDSDEs with Lévy process for a fixed terminal time under Lipschitzian assumption. This section can be viewed as a continuation of such a theme, and will exploit the above theory of BDSDEP with non-Lipschitzian coefficients and random terminal time in order to provide a probabilistic formula for the solution of a quasilinear SPDIE.

Let $D$ be a bound domain in $\mathbb{R}^{m}$ with boundary $\partial D=S$.

First, consider the following forward SDE with Poisson jumps in $\mathbb{R}^{m}$ for any given $(t, x) \in[0, T] \times D$

$$
\begin{aligned}
X_{t}= & x+\int_{t}^{s} b\left(r, X_{r}\right) d r+\int_{t}^{s} g\left(r, X_{r}\right) d W_{r} \\
& +\int_{t}^{s} \int_{Z} h\left(r_{-}, X_{r_{-}}, z\right) \tilde{N}(d z \mathrm{~d} r), \quad t \leq s \leq T,
\end{aligned}
$$

where

$$
b:[0, T] \times \mathbb{R}^{m} \rightarrow \mathbb{R}^{m}, \sigma:[0, T] \times \mathbb{R}^{m} \rightarrow \mathbb{R}^{m \times d}, h:[0, T] \times \mathbb{R}^{m} \times Z \rightarrow \mathbb{R}^{m} .
$$

It is known that, if coefficients are less than linear increasing, and satisfy the Lipschitz condition, then SDE (5) has a unique solution. (See [12])

Now for any $(t, x) \in[0, T] \times D$, let

$$
\tau=\tau_{x}=\inf \left\{s>t: X_{s}^{t, x} \notin D\right\}, \text { and } \tau=\tau_{x}=T, \text { for } \inf \{\phi\} .
$$

Consider the following BDSDEP (for simplicity, denote $X_{s}=X_{s}^{t, x}$ ),

$$
\begin{aligned}
P_{s}= & \Phi\left(X_{\tau}\right)+\int_{s \wedge \tau}^{\tau} f\left(r, X_{r}, P_{r}, Q_{r}, K_{r}\right) d r+\int_{s \wedge \tau}^{\tau} g\left(r, X_{r}, P_{r}, Q_{r}, K_{r}\right) d B_{r} \\
& +\int_{s \wedge \tau}^{\tau} Q_{r} d W_{r}+\int_{s \wedge \tau}^{\tau} \int_{Z} K_{r_{-}}(z) \tilde{N}(d z d r), \quad t \leq s \leq T,
\end{aligned}
$$

where

$$
\begin{aligned}
& f:[0, T] \times \mathbb{R}^{m} \times \mathbb{R}^{n} \times \mathbb{R}^{n \times d} \times L_{\lambda(\cdot)}^{2}\left(\mathbb{R}^{n}\right) \rightarrow \mathbb{R}^{n}, \\
& g:[0, T] \times \mathbb{R}^{m} \times \mathbb{R}^{n} \times \mathbb{R}^{n \times d} \times L_{\lambda(\cdot)}^{2}\left(\mathbb{R}^{n}\right) \rightarrow \mathbb{R}^{n \times l}, \\
& \Phi: \mathbb{R}^{m} \rightarrow \mathbb{R}^{n} .
\end{aligned}
$$

Suppose that $f(t, x, \cdot, \cdot, \cdot)$ and $g(t, x, \cdot, \cdot, \cdot)$ satisfy the conditions in Theorem 3.4 uniformly for $t$ and $x$, and suppose that $E\left|\Phi\left(X_{\tau}\right)\right|^{2}<\infty$, then by Theorem 3.4, $\operatorname{BDSDEP}($ (6) $)$ has a unique solution $\left(P_{t}, Q_{t}, K_{t}\right) \in S^{2}\left([0, \tau] ; \mathbb{R}^{n}\right) \times M^{2}\left(0, \tau ; \mathbb{R}^{n \times d}\right)$ $\times F_{N}^{2}\left(0, \tau ; \mathbb{R}^{n}\right)$. 
We now relate BDSDEP (6) to the following system of quasilinear secondorder parabolic SPDIE:

$$
\left\{\begin{array}{l}
\mathcal{L} u(t, x) d t \\
=f(t, x, u(t, x), \nabla u(t, x) g(t, x), u(t, x+h(t, x, \cdot))-u(t, x)) d t \\
+g(t, x, u(t, x), \nabla u(t, x) g(t, x), u(t, x+h(t, x, \cdot))-u(t, x)) d B_{t}, \\
\forall(t, x) \in[0, T] \times D \\
u(T, x)=\Phi(x), \quad \forall(t, x) \in[0, T] \times D \\
u(t, x)=\Phi(x), \quad \forall(t, x) \in[0, T] \times S,
\end{array}\right.
$$

where $u: \mathbb{R}_{+} \times \mathbb{R}^{m} \rightarrow \mathbb{R}^{n}$,

$$
\mathcal{L} u=\left(\begin{array}{c}
L u_{1} \\
\vdots \\
L u_{n}
\end{array}\right)
$$

with

$$
\begin{aligned}
& L u_{k}(t, x) \\
= & \frac{\partial u_{k}}{\partial t}(t, x)+\sum_{i=1}^{n} b_{i}(t, x) \frac{\partial u_{k}}{\partial x_{i}}(t, x)+\frac{1}{2} \sum_{i, j=1}^{n}(\sigma \sigma *)_{i j}(t, x) \frac{\partial^{2} u_{k}}{\partial x_{i} \partial x_{j}}(t, x) \\
& +\int_{Z}\left(u_{k}(t, x+h(t, x, z))-u_{k}(t, x)-\sum_{i=1}^{n} h_{i}(t, x, z) \frac{\partial u_{k}}{\partial x_{i}}(t, x)\right) \lambda(d z), \\
& k=1, \cdots, n .
\end{aligned}
$$

Now assume that $\sigma$ is uniformly non-degenerate, i.e. there exists a constant $\beta>0$, such that

$$
\frac{1}{2} \sum_{i, j=1}^{n}(\sigma \sigma *)_{i j}(t, x) \xi_{i} \xi_{j} \geq \beta|\xi|^{2}, \text { for all } \xi \in \mathbb{R}^{m}, \text { and }(t, x) \in[0, T] \times \bar{D},
$$

where $\bar{D}=$ the closure of $D$. Hence, SPDIE (7) is a true quasilinear type equation.

We can assert that

Theorem 5.1 Under the above related conditions, and $b, \sigma, h, f$ and $g$ are of class $C^{3}$, and $\Phi$ is of class $C^{2}$. Suppose SPDIE (7) has a unique solution $u(t, x) \in C^{1,2}\left(\Omega \times[0, T] \times \mathbb{R}^{m} ; \mathbb{R}^{n}\right)$. Then, for any given $(t, x), u(t, x)$ has the following interpretation

$$
u(t, x)=P_{t},
$$

where $P_{t}$ is determined uniquely by (5) and (6).

Proof Applying Itô's formula to $u\left(t, X_{t}\right)$ (see Theorem 6 in [12]) on $[s \wedge \tau, \tau]$, we 
obtain

$$
\begin{aligned}
& u\left(\tau, X_{\tau}\right)-u\left(s \wedge \tau, X_{s \wedge \tau}\right) \\
= & \int_{s \wedge \tau}^{\tau} \frac{\partial u}{\partial r}\left(r, X_{r}\right) d r+\int_{s \wedge \tau}^{\tau} \sum_{i=1}^{m} b_{i}\left(r, X_{r}\right) \frac{\partial u}{\partial x_{i}}\left(r, X_{r}\right) d r \\
& +\int_{s \wedge \tau}^{\tau} \nabla u\left(r, X_{r}\right) \sigma\left(r, X_{r}\right) d W_{r}+\int_{s \wedge \tau}^{\tau} \frac{1}{2} \sum_{i, j=1}^{m}(\sigma \sigma *)_{i j}\left(r, X_{r}\right) \frac{\partial^{2} u}{\partial x_{i} \partial x_{j}}\left(r, X_{r}\right) d r \\
& +\int_{s \wedge \tau}^{\tau} \int_{Z}\left(u\left(r, X_{r}+h\left(r, X_{r}, z\right)\right)-u\left(r, X_{r}\right)\right) \tilde{N}(d z d r) \\
& +\int_{s \wedge \tau}^{\tau} \int_{Z}\left(u\left(r, X_{r}+h\left(r, X_{r}, z\right)\right)-u\left(r, X_{r}\right)\right. \\
& \left.-\sum_{i=1}^{m} h_{i}\left(s, X_{s}, z\right) \frac{\partial u}{\partial x_{i}}\left(r, X_{r}\right)\right) \lambda(d z) d r .
\end{aligned}
$$

Because $u(t, x)$ satisfies SPDIE (7), it holds that

$$
\begin{aligned}
& \Phi\left(X_{\tau}\right)-u\left(s \wedge \tau, X_{s \wedge \tau}\right) \\
= & \int_{s \wedge \tau}^{\tau} f\left(r, X_{r}, u\left(r, X_{r}\right), \nabla u\left(r, X_{r}\right) \sigma\left(r, X_{r}, u\left(r, X_{r}\right)\right),\right. \\
& \left.u\left(r, X_{r}+h\left(r, X_{r}, \cdot\right)\right)-u\left(r, X_{r}\right)\right) d r \\
& +\int_{s \wedge \tau}^{\tau} g\left(r, X_{r}, u\left(r, X_{r}\right), \nabla u\left(r, X_{r}\right) \sigma\left(r, X_{r}, u\left(r, X_{r}\right)\right),\right. \\
& \left.u\left(r, X_{r}+h\left(r, X_{r}, \cdot\right)\right)-u\left(r, X_{r}\right)\right) d B_{r}+\int_{s \wedge \tau}^{\tau} \nabla u\left(r, X_{r}\right) g\left(r, X_{r}\right) d W_{r} \\
& +\int_{s \wedge \tau}^{\tau} \int_{Z}\left(u\left(r, X_{r}+h\left(r, X_{r}, z\right)\right)-u\left(r, X_{r}\right)\right) \tilde{N}(d z d r) .
\end{aligned}
$$

It is easy to check that $\left(u\left(t, X_{t}\right), \nabla u\left(t, X_{t}\right) \sigma\left(t, X_{t}\right), u\left(t, X_{t}+h\left(t, X_{t}, \cdot\right)\right)-u\left(t, X_{t}\right)\right)$ coincides with the unique solution of BDSDEP (6). It follows that

$$
u(t, x)=P_{t} .
$$

Remark 5.2 (8) can be called a stochastic Feynman-Kac formula for SPDIE (7), which is a useful tool in the study of SPDIE.

\section{References}

[1] V. Bally, A. Matoussi, Weak solutions for SPDEs and backward doubly stochastic differential equations, J. Theoret. Probab. 14 (2001) 125-164.

[2] G. Barles, R. Buckdahn, E. Pardoux, Backward stochastic differential equations and integral-partial differential equations, Stoch. Stoch. Rep. 60 (1997) 57-83.

[3] R. Darling, E. Pardoux, Backward SDE with random terminal time and applications to semilinear elliptic PDE, Ann. Probab. 25 (1997) 1135-1159. 
[4] D. Duffie, L. Epstein, Stochastic differential utilities, Econometrica 60 (1992) 354-439.

[5] L. Hu, Y. Ren, Stochastic PDIEs with nonlinear Neumann boundary conditions and generalized backward doubly stochastic differential equations driven by Lévy processes, J. Comput. Appl. Math. 229 (2009) 230-239.

[6] N. Ikeda, S. Watanabe, "Stochastic differential equations and diffusion process", North Holland Publishing Company, Amsterdam, 1981.

[7] E. Pardoux, S. Peng, Adapted solution of a backward stochastic differential equation, Systems Control Lett. 14 (1990) 55-61.

[8] E. Pardoux, S. Peng, Backward doubly stochastic differential equations and systems of quasilinear parabolic SPDEs, Probab. Theory Related Fields 98 (1994) 209-227.

[9] S. Peng, Probabilistic interpretation for systems of quasilinear parabolic partial differential equations, Stoch. Stoch. Rep. 37 (1991) 61-74.

[10] Y. Ren, A. Lin, L. Hu, Stochastic PDIEs and backward doubly stochastic differential equations driven by Lévy processes, J. Comput. Appl. Math. 223 (2009) 901-907.

[11] Y. Shi, Y. Gu, K. Liu, Comparison theorems of backward doubly stochastic differential equations and applications, Stoch. Anal. Appl. 23 (2005), 97-110.

[12] R. Situ, On solution of backward stochastic differential equations with jumps and applications, Stoch. Process. Appl. 66 (1997) 209-236.

[13] X. Sun, Y. Lu, The property for solutions of the multi-dimensional backward doubly stochastic differential equations with jumps, Chin. J. Appl. Probab. Stat. 24 (2008) 73-82.

[14] S. Tang, X. Li, Necessary condition for optional control of stochastic system with random jumps, SIAM J. Control Optim. 32 (1994) 1447-1475.

[15] J. Yin, X. Mao, The adapted solution and comparison theorem for backward stochastic differential equations with Poisson jumps and applications, J. Math. Anal. Appl. 346 (2008) $345-358$.

[16] Q. Zhang, H. Zhao, Stationary solutions of SPDEs and infinite horizon BDSDEs, J. Funct. Anal. 252 (2007) 171-219. 